\title{
Primary Basidiobolomycosis of the Cecum in Immunocompetent Patients: Two Case Reports
}

\author{
Mahmoud R.A. Hussein a, Abdullah S. Alqahtani ${ }^{\text {b, g, Yahia Alhagawic }}{ }^{c}$ Naif S. Al Saglan ${ }^{\text {, }}$ \\ Abdullah M. Albishic ${ }^{\mathrm{c}}$ Feras O. Ahmed ${ }^{\mathrm{b}}$, Mohammad E. Rabie ${ }^{\mathrm{b}}$, \\ Mohamed F. Bazeed ${ }^{e}$, Eman E. Abu-Dief ${ }^{f}$
}

\begin{abstract}
Colonic basidiobolomycosis is a rare fungal infection caused by Basidiobolus ranarum. Primary cecal basidiobolomycosis is an exceptionally rare condition. The study describes two cases of primary basidiobolomycosis of the cecum in immunocompetent male and female patients (one each). The patients presented with fever, abdominal pain, weight loss, eosinophilia, and high erythrocyte sedimentation rates. Computed tomography revealed wall thickening and mass lesions involving the cecum, suggesting malignancy. Right hemicolectomies were performed to relieve the intestinal obstruction. On microscopy, there were destructive, transmural eosinophil-rich pyogranulomatous reactions with thinwalled, pauci-septated fungal elements surrounded by Splendore-Hoeppli bodies. The patients received antifungal drugs, with no evidence of dissemination or recurrence on follow-up. Primary cecal basidiobolomycosis in immunocompetent hosts is a rare occurrence. It oftentimes clinically masquerades malignant neoplasms and therefore its identification mandates its inclusion in the differential diagnosis of a colonic mass, equally both on the part of the clinicians and pathologists.
\end{abstract}

Keywords: Basidiobolomycosis; Fungal; Cecum; Mass; SplendoreHoeppli bodies

\section{Introduction}

There are more than100,000 fungi worldwide, but only about

Manuscript submitted February 5, 2021, accepted February 25, 2021

Published online April 21, 2021

aDepartment of Pathology, Faculty of Medicine, Assiut University, Assiut, Egypt bDepartment of General Surgery, Armed Forces Hospital, Southern Region, Saudi Arabia

'Department of Gastroenterology, Armed Forces Hospital, Southern Region, Saudi Arabia

${ }^{\mathrm{d}}$ Department of Infectious Diseases, Armed Forces Hospital, Southern Region, Saudi Arabia

eDepartment of Radiology, Armed Forces Hospital, Southern Region, Saudi Arabia fDepartment of Histology, Faculty of Medicine, Sohag and Merit Universities, Sohag, Egypt

'Corresponding Author: Abdullah S. Alqahtani, Department of General Surgery, Armed forces Hospitals, Southern Region, Saudi Arabia.

Email: dr_asq@yahoo.com

doi: https://doi.org/10.14740/gr1368
150 of them are pathogenic. Basidiobolomycosis is caused by the fungi of Basidiobolus ranarum (B. ranarum), which is a low virulent fungus of the order Entomophthorales that belongs to the class Zygomycetes. B. ranarum fungi were first reported in frogs in 1886 . They are environmental saprophytic fungi found in decaying fruits, vegetable matters and soil. They are commensals in the guts of bats, dogs, frogs, amphibians, fish and reptiles [1-4].

In humans, basidiobolomycosis is an aggressive and fatal disease. Its treatment options include surgical resection and intensive, and prolonged systemic antifungal treatment. Basidiobolomycosis, not like other fungal infections, always impacts immunocompetent individuals. Infections of $B$. ranarum are well-known in the skin and subcutaneous tissue; however, gastrointestinal basidiobolomycosis (GIB) is a rare occurrence $[2,5,6]$. In humans, the first identified case of subcutaneous basidiobolomycosis infection was documented in Indonesia in 1956 [7]. In 1964, the initially recognized case of GIB was reported in a 4-year-old boy [8]. Here, in this endeavor, the authors presented a reappraisal of the current relevant literature about basidiobolomycosis. It also describes two cases of primary basidiobolomycosis of the cecum.

In zygomycosis (phycomycosis), there is the infiltration of the tissues by broad, non-septate, fungal hyphae. The latter includes multiple genera such as Mucorales, Rhizopus, Rhizomucor, Conidiobolus, Absidia, and Basidiobolus [2]. Two orders are included in Zygomycetes namely Mucorales and Entomophthorales. Mucorales involve only the immunocompromised patient. Just the opposite, Entomophthorales, which include Basidiobolus genera, involves usually the immunocompetent individuals, and it is exceptionally rare in immunocompromised patients. Just the opposite, Mucormycosis has been rarely reported in immunocompetent hosts with no underlying disease. The laboratory identification of Zygomycetes is established on morphological features, namely the presence of either sexual reproductive elements (known as zygospores) or asexual reproductive bodies (known as conidia) [9, 10].

Primary basidiobolomycosis of the colon is an exceptionally rare pathology that can affect all age groups. It is endemic in warm areas such as tropical and subtropical regions of Latin America, Arizona, Asia (Saudi Arabia, Kuwait, Iran, and India), and Africa because the fungus prefers to flourish mostly in a warm and humid atmosphere [11]. The clinical presentation includes fever, weight loss, abdominal pain in a patient with intestinal mass or wall thickening, and concomitant el- 

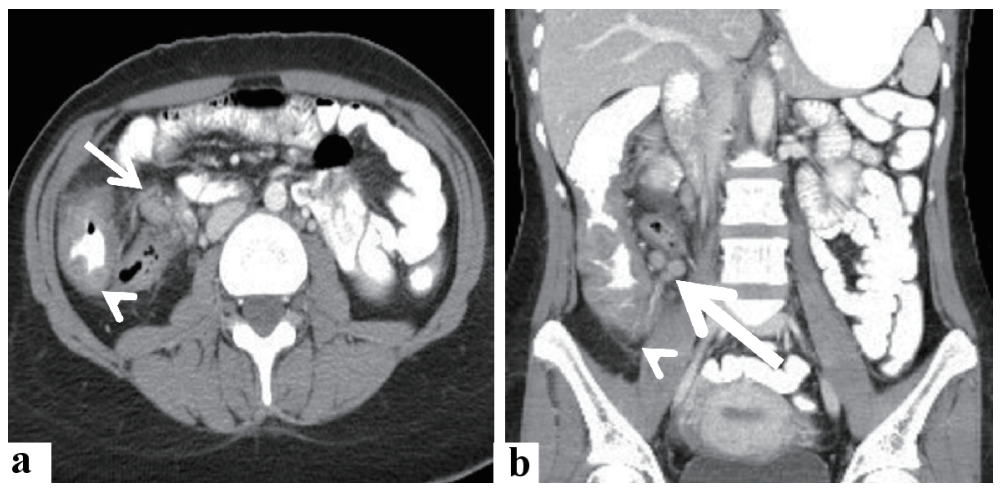

Figure 1. Radiological features of the cecal and lymph nodal basidiobolomycosis (case 1). Abdominal CT scan with intravenous and oral contrast (a) axial, and (b) coronal revealed that diffuse enhancing soft tissue thickening (arrowhead) is seen involving cecum, ascending colon, and an appendix with enlarged enhancing local lymph nodes (arrow). CT: computed tomography.

evated erythrocyte sedimentation rate (ESR) and eosinophilia $[2,8,12-15]$. The involvement of the cecum is rare and can masquerade several conditions including ulcerative colitis, Crohn's disease, indeterminate colitis, immunodeficiency disorder, or malignant neoplasms (colonic lymphomas, carcinomas, sarcomas), and as such, it poses a diagnostic challenge [16]. Failure of response to treatment regimens consisting of anti-inflammatory drugs, immunosuppressive therapy, and immune-modulatory agents used in the management of inflammatory bowel diseases should raise the suspicion of an alternative cause for eosinophil-rich inflammation such as basidiobolomycosis [6]. The management options in basidiobolomycosis include combined surgical resection and antifungal drugs such as itraconazole and amphotericin [17].

Here, this study describes two cases of primary basidiobolomycosis of the cecum. The unique addition of our study is that these cases were reported in immunocompetent patients and both presented with features masquerading colon cancer. Our study also emphasized that the best diagnostic clue for colonic basidiobolomycosis is by including this entity in the differential diagnosis, especially in immunocompetent patients presenting with nonspecific clinical (unexplained fever and abdominal pain), radiological (colonic wall thickening or mass lesions), and laboratory (eosinophilia, and high ESR) findings.

\section{Cases Reports}

\section{Case 1}

A 23-year male presented with a history of constipation, weight loss, and rectal bleeding. He had no history of immune deficiency or immunosuppressive therapy. There was no evidence of lymphadenopathy or organomegaly on general examination. The white blood cell (WBC) counts were $6.4 \times$ 10\%/L, eosinophils: $19.8 \%$, and absolute eosinophil count: 1.26 $\times 10^{9}$ cells/L. Other laboratory investigations were unremarkable. Computed tomography (CT) of the abdomen and pelvis with oral and intravenous contrast revealed a large soft tissue density mass measuring $10.5 \times 8.8 \times 4.2 \mathrm{~cm}$ (in its maximal dimensions) at the anatomical location of the cecum and ascend- ing colon, contacting the abdominal wall anteriorly, and psoas muscle posteriorly. The appendix could not be visualized. The mass was associated with mild amounts of fluid $(4.4 \times 1.9 \times$ $2.7 \mathrm{~cm}$ ) and multiple variable-sized localized lymph nodes (the smallest was $0.5 \mathrm{~cm}$ and the largest was up to $2.0 \mathrm{~cm}$ ). No CT findings of intestinal obstruction were noted. The lung, kidneys, adrenals, liver, spleen, and pancreas were unremarkable. A summary of the radiological findings is shown in Figure 1.

The radiological impressions included the neoplastic versus inflammatory process. Colonoscopic examination revealed a long-segment ulcerative lesion involving the cecum and extending into the proximal part of the ascending colon. Histological examination of colonoscopic biopsy specimens from the ulcerative lesion revealed features of eosinophil-rich colitis with fungal elements in the expanded lamina propria (Fig. 2). En bloc resection of the terminal ileum, appendix, cecum, and right colon was done. At the time of laparotomy, a huge heterogeneous mass was seen involving the cecum and extending to the proximal part of the right colon. The regional lymph nodes were markedly enlarged, varying in size from 1.0 to 3.0 $\mathrm{cm}$ with a pink cut section. The terminal ileum was grossly unremarkable. Microbiological analysis was not contributory. Histologically, there was an eosinophil-rich granulomatous reaction that involved the mucosa, submucosa, and muscle layers with dense eosinophil-rich mixed inflammatory cell infiltrate composed of lymphocytes, histiocytes, and neutrophils, and multinucleated giant cells containing fungal elements. Periodic acid-Schiff (PAS) stains revealed fungal elements in the form of predominantly non-septate, broad, thick-walled, pleomorphic hyphae, with irregular, non-parallel contours and occasional haphazard branching. Three lymph nodes showed fungal hyphae and spore amid eosinophil-rich pyogranulomatous reaction. A summary of the histological findings is shown in Figure 3. Panfungal polymerase chain reaction (PCR) performed on DNA extracted directly from a tissue sample confirmed the presence of $18 \mathrm{~S}$ rRNA genes of fungal pathogens using the UMD test system (Lot NO.B24rKU010.048, Mozyme $\mathrm{GmbH} \& \mathrm{CO} \mathrm{KG}$ ). The diagnosis of basidiobolomycosis was established. The treatment regimen included the use of itraconazole $200 \mathrm{mg}$ tablet twice daily for 6 months. Follow-up of the patient revealed a good response to the treatment. No evidence 

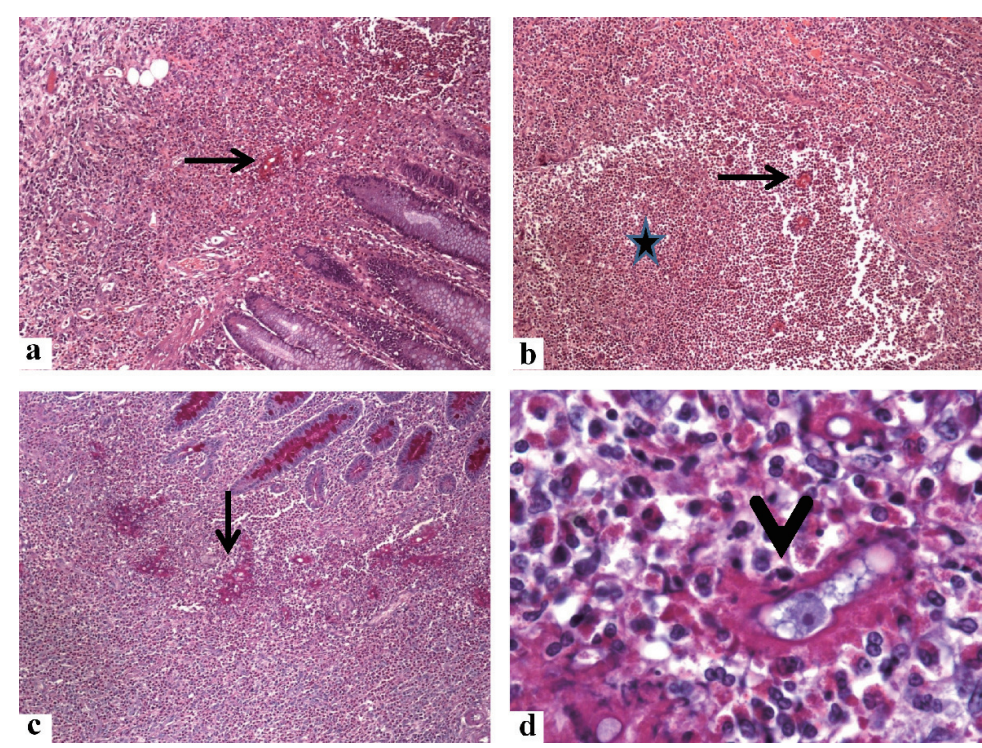

Figure 2. Histological findings in the colonoscopic biopsy specimen from the cecal basidiobolomycosis (case 1). Sections from the cecal ulcerative mass showing some fungal hyphae (arrows) amid dense eosinophil-rich mixed inflammatory cell infiltrate and eosinophilic microabscess (star) in the lamina propria. The B. ranarum appears as hyphae with zygospores. The zygospore resembles trophozoites of amoeba, has foamy cytoplasm with a large nucleolus (arrowhead). The original magnifications are: a, b, c: $\times 100$, and d: $\times 1,000)$. B. ranarum: Basidiobolus ranarum.

of disease recurrence in the 6 months' follow-up period.

\section{Case 2}

A 33-year-old female patient had a long history of vague abdominal pain and loss of weight. The WBC was $26.5 \times 10^{9}$ cells/L, eosinophils: $19 \%$, and absolute eosinophil count: $5 \times$ $10^{9}$ cells/L. Colonoscopic examination revealed a long segment of fungating mass at the cecum and the proximal part of the right colon with ulceration and hard mucosa. A summary of the colonoscopic findings is shown in Figure 4. CT of the abdomen and pelvis with oral and intravenous contrast revealed mural thickening involving the cecum and the ascending colon for about $10 \mathrm{~cm}$. The mural thickening was also seen involving the appendix (measuring $1.8 \mathrm{~cm}$ in diameter) which is seen extending cranially. There were few associated localized lymph nodes, the largest was measuring about $1.4 \mathrm{~cm}$ in diameter. No $\mathrm{CT}$ findings of intestinal obstruction were noted. A summary of the radiological findings was depicted in Figure 5. Multiple biopsies were obtained, and the patient was referred for a general surgery consultation.

Histologically, there was dense eosinophil-rich mixed inflammatory cell infiltrate with occasional PAS-positive fungal structures including non-septate, broad, thick-walled, variablesized hyphae, consistent with the diagnosis of basidiobolomycosis. Other findings included epithelioid cell granulomas, and active colitis in the form of cryptitis, and crypt abscess (Fig. 6). Suddenly, the patient presented to the Department of Surgery with manifestations of intestinal obstruction in the form of sudden acute abdominal pain, nausea, and vomiting. Intraoperatively, a huge heterogeneous mass was seen involving the cecum and extending to the proximal part of the right colon.
The appendix (measuring $7 \mathrm{~cm}$ in length and $2 \mathrm{~cm}$ in diameter) showed diffuse thickening of the wall and obliteration of the lumen. The terminal ileum was grossly unremarkable. En bloc resection of the terminal ileum, appendix, cecum, and ascending colon was performed. Microbiological analysis (culture of the peritoneal fluid) was negative. Molecular studies (panfungal PCR) were done using DNA extracted from the lesional colonic tissue and they detected 18S rRNA genes using the UMD test system (Lot NO.B24rKU010.048, Mozyme GmbH \& $\mathrm{CO} \mathrm{KG})$.

Histologically, sections from the appendix and cecum showed a granulomatous reaction that involved the mucosa, submucosa, and muscle layers with dense eosinophil-rich mixed inflammatory cell infiltrate composed of lymphocytes, histiocytes, and neutrophils and multinucleate giant cells containing fungal elements. PAS stains revealed fungal elements in the form of predominantly non-septate, broad, thick-walled, pleomorphic hyphae, with irregular, non-parallel contours and occasional haphazard branching. A summary of the histological findings was presented in Figure 7. Panfungal PCR performed on DNA extracted directly from a tissue sample confirmed the presence of Basidiobolus. The diagnosis of basidiobolomycosis has been established. The treatment regimen included the use of itraconazole $200 \mathrm{mg}$ tablet twice daily for 6 months. Follow-up of the patient revealed a good response to the treatment. No evidence of disease recurrence in the 8 months' follow-up period.

\section{Discussion}

GIB is acquired in most cases by ingestion of contaminated water, or food including fruits, and vegetables contaminated 

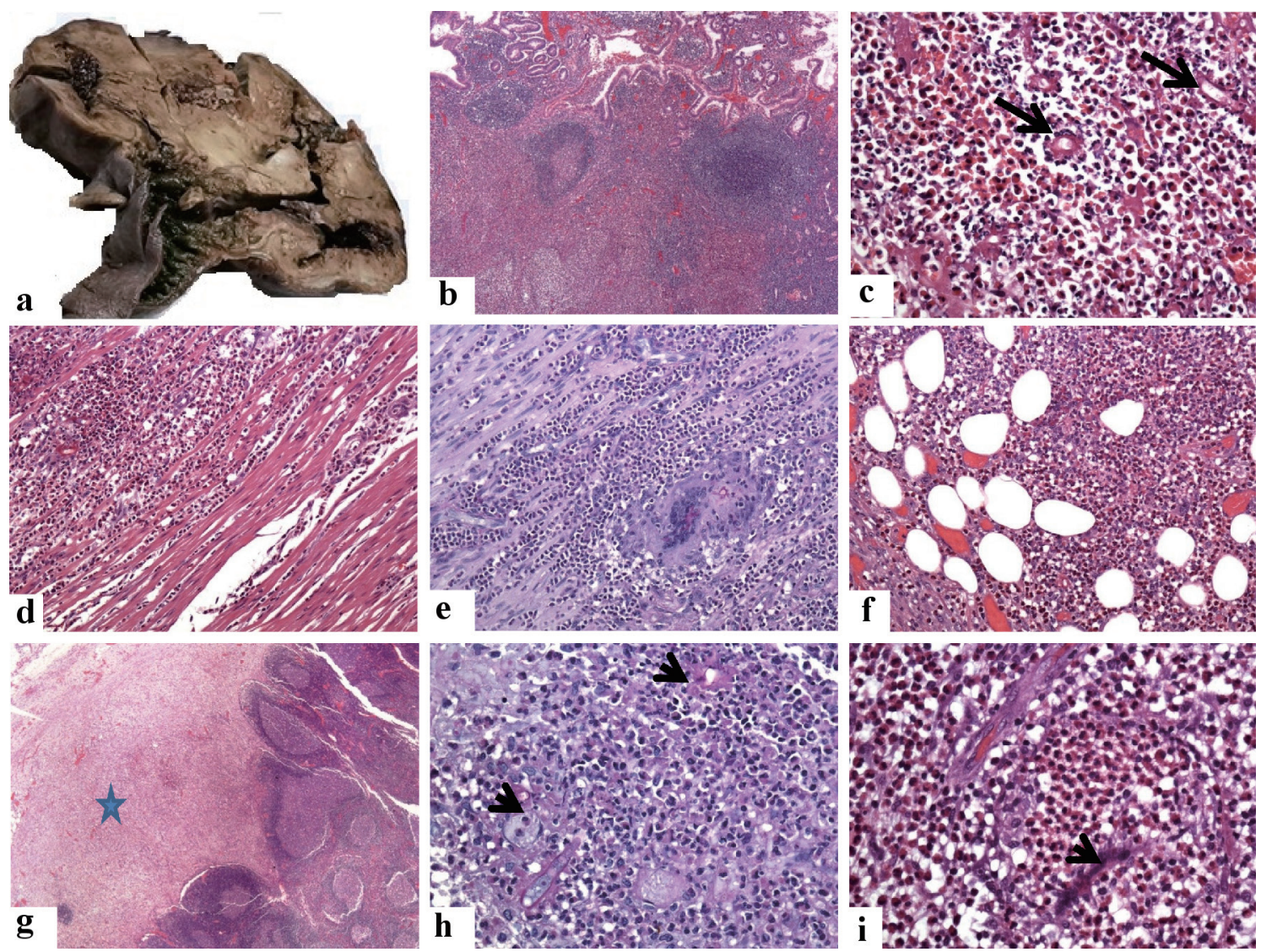

Figure 3. Pathological features of the cecal and lymph nodal basidiobolomycosis (case 1). (a) Grossly, there is diffuse thickening of the wall of the cecum and proximal part of the ascending colon. They are involved by a huge firm mass with a grayish cut section. (b-f) Sections from the cecal mass showing ulceration of the mucosa, increased density of eosinophil-rich mixed inflammatory cell infiltrate in the lamina propria with the formation of multiple lymphoid follicles, around fungal hyphae (arrows, the original magnifications are b: $\times 40$, and c: $\times 400$ ). This eosinophil-rich mixed inflammation destroys the muscularis propria (d: $\times 200$ ) with the formation of multinucleated giant cells, phagocytosing the fungal elements (PAS stain, e: $\times 200$ ), and extending into the subserosal fatty tissue $\mathrm{f}: \times 200)$. ( $\mathrm{g}-\mathrm{i})$ Sections show replacement of the subcapsular sinuses $($ star, $\mathrm{g}: \times 20)$ of the regional lymph nodes by and multiple fungal structures (PAS stain, arrow, h: $\times 400)$. The zygospores resemble trophozoites of amoeba, have foamy cytoplasm with a large nucleolus but no heterochromatin (i: $\times 1,000$, arrowhead), amid eosinophilic microabscess. PAS: Periodic acid-Schiff.

by animal fecal matters. Other proposed routes include using contaminated papers for cleaning the skin (e.g., toilet papers for cleaning of the skin after defecation), implantation of the fungus following surgical procedures. The intake of ranitidine can reduce gastric acidity and therefore allows for the survival of the fungi after gastric passing. The fungi of $B$. ranarum are commonly found in the soil and therefore gardeners, farmers, and landscapers are more exposed to GIB [11]. Moreover, cigarette smoking can decrease mucosal WBC function and facilitate fungal infection by $B$. ranarum $[12,18]$. The pathogenesis of primary GIB is poorly understood. It may result from local insults to the colonic mucosa, with the breakdown in the mucosal defense barrier and immunity. Several host defense mechanisms combat zygomycotic infection. They include sequestration of iron molecules, generation of the reactive oxidative radicles, and metabolites that destroy the fungal structures. Moreover, the polymorphonuclear leukocytes and histiocytes can kill the fungal bodies by releasing defensins that represent strong cationic peptides. The development of zygomycosis depends on the scavenging iron molecules necessary for fungal growth from the host lesional tissues. The fungal elements also secure access to the host blood vessels resulting in the dissemination of the fungal structures [19].

The diagnosis of basidiobolomycosis depends on the detection of B. ranarum. Culture on Sabouraud agar at $20-30{ }^{\circ} \mathrm{C}$ with incubation for 2 - 3 days is the gold standard for definitive diagnosis. The fungal growth appears as white to pale grey, waxy, or glabrous colonies with radial folds covered by aerial hyphae [20]. However, in most reported cases, the patients have undergone surgical resection with the clinical impression of mass and therefore the specimens have been transferred to the laboratories in formalin and no cultures have been performed.

On histology, B. ranarum appears as thin-walled, pauci- 


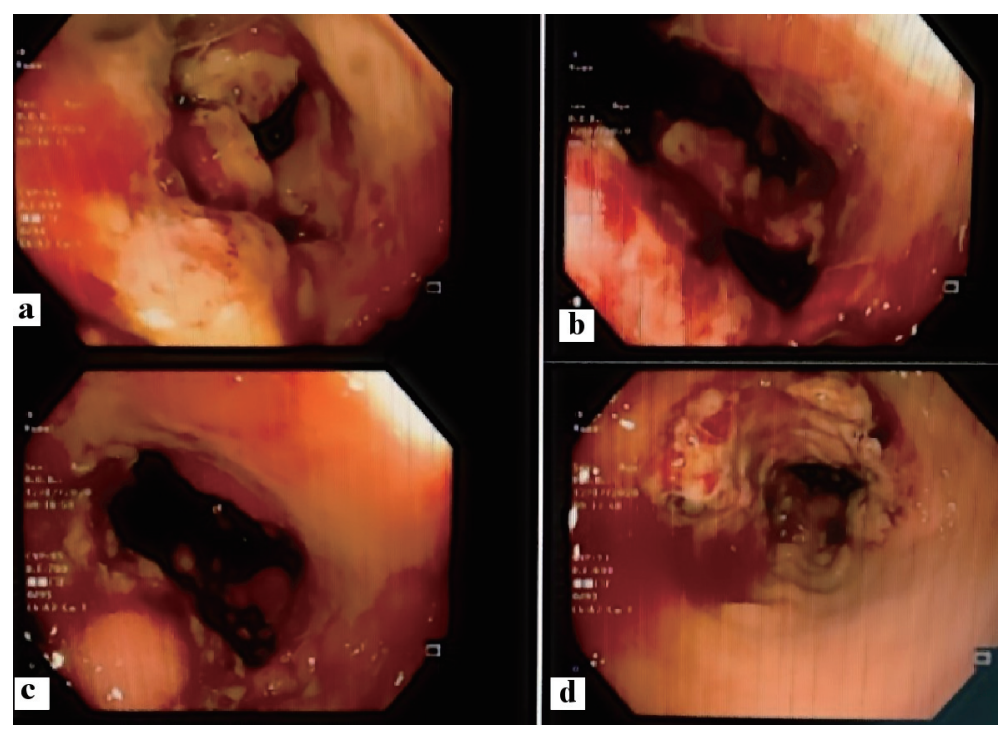

Figure 4. Colonoscopic features of the cecal and appendicular basidiobolomycosis (case 2): (a) Ulcerative mass obstructing the cecum, (b) bleeding ulcer, (c) polypoidal lesion, and (d) narrowing of the colonic lumen.

septated hyphae admixed with zygospores. The hyphae (mean of the hyphal diameter, $9 \mu \mathrm{m}$; ranging in diameter from 5.0 $\mu \mathrm{m}$ up to $20 \mu \mathrm{m})$ are surrounded by thick radiating, eosinophilic, sleeve-like $(2.5-25 \mu \mathrm{m})$ amorphous hyalinized sub- stances (Splendor-Hopllie phenomenon). The presence of these eosinophilic sleeves can differentiate zygomycosis from pythiosis. In the latter, the eosinophilic sleeves are either completely absent or very thin. The zygospores resemble trophozo-
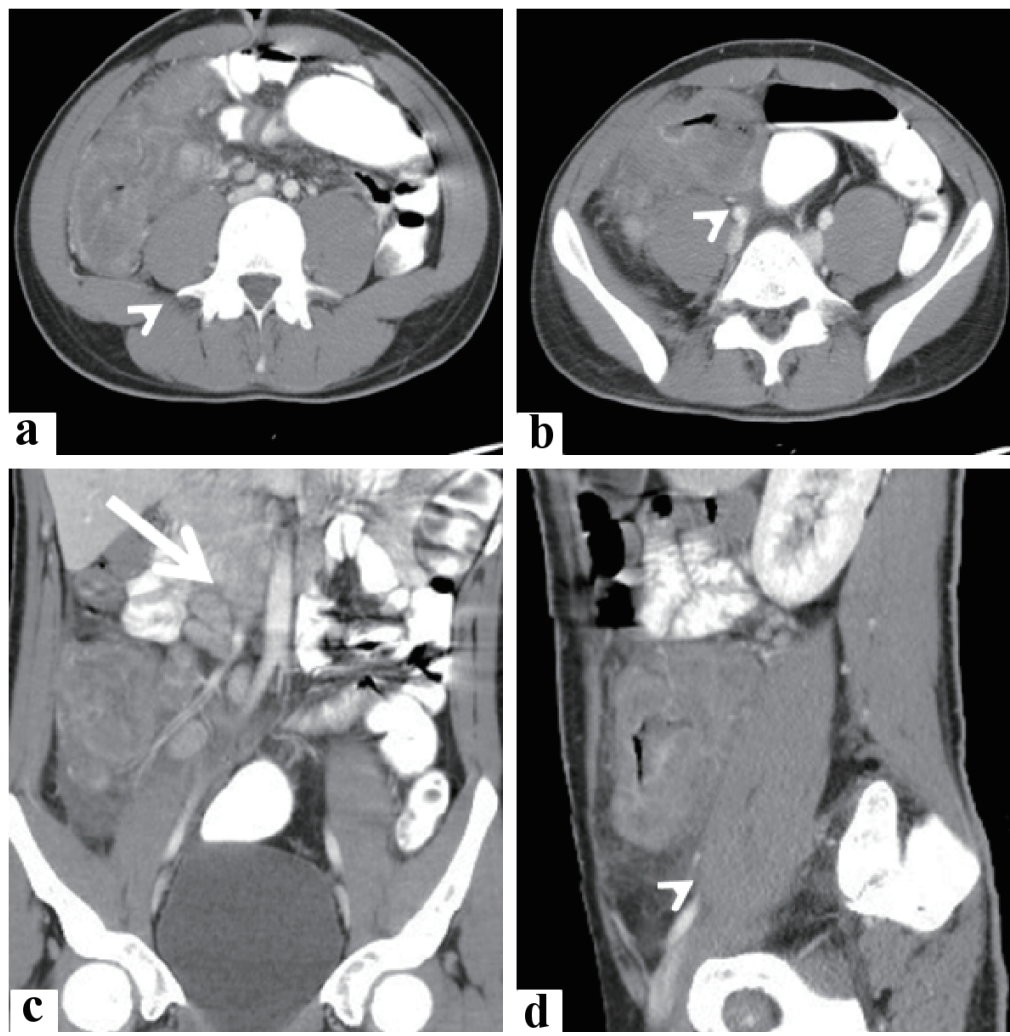

Figure 5. Radiological features of the cecal and appendicular basidiobolomycosis (case 2). Abdominal CT scan with intravenous and oral contrast (a, b) axial, (c) coronal, and (d) sagittal revealed: lobulated enhancing soft tissue mass lesion (arrowhead) is seen involving cecum and ascending colon, contacting abdominal wall and right psoas muscle. It is seen associated with minimal local fluid and enlarged enhancing mesenteric lymph nodes (arrow). CT: computed tomography. 

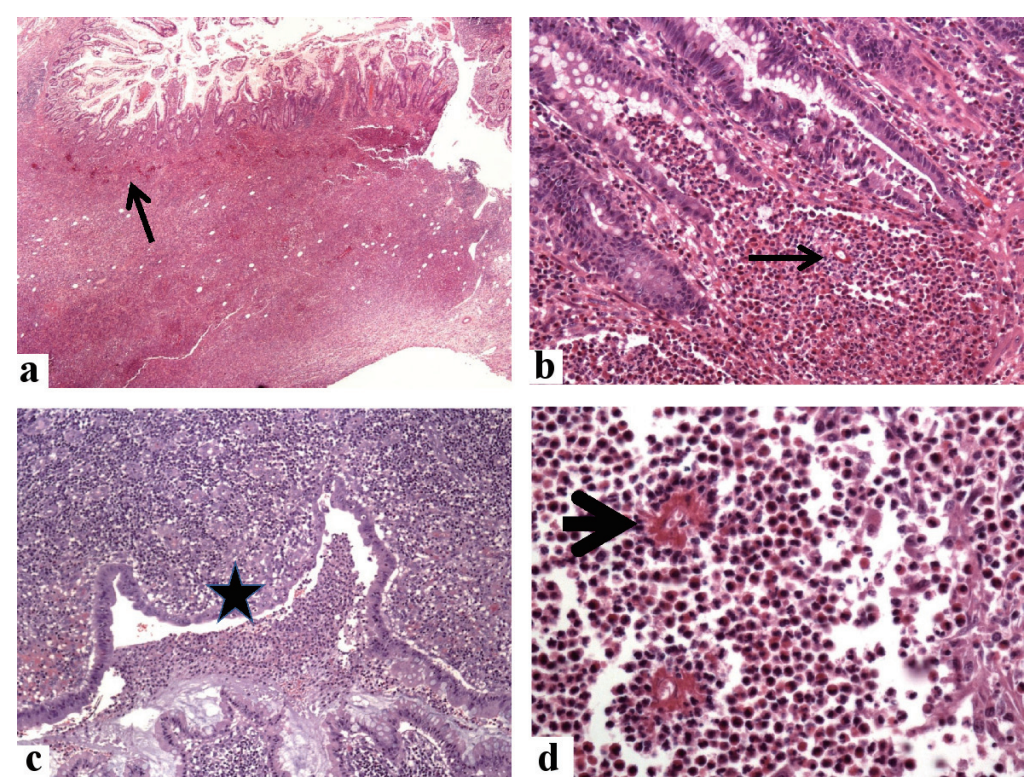

Figure 6. Histological findings in the colonoscopic biopsy specimen from the cecal and appendicular basidiobolomycosis (case 2). Sections from the cecal mass show expansion of the lamina propria by mixed eosinophil-rich inflammatory cells with multiple fungal elements (arrows, hematoxylin and eosin (H\&E) stain, a: $\times 20$, and b: $\times 200$ ), mucosal erosions and luminal necrotic materials with polymorphs (star, c: $\times 200$ ), and fungal hyphae surrounded by radiating eosinophilic Splendore-Hoeppli bodies (arrowhead, d: × 400).

ites of amoeba, have foamy cytoplasm with a large nucleolus but no heterochromatin [2]. The fungal stains PAS/(periodic acid-Schiff/diastase) PASD and Gomori methenamine silver (GMS) can intensify the staining of the fungal walls. The organisms are surrounded by eosinophil-rich pyogranulomatous inflammation with giant cells, a reaction pattern that is oftentimes mistaken for granulomatous colitis (Crohn's disease, amebiasis, and tuberculosis) [21]. This histological finding is very characteristic of $B$. ranarum. Basidiobolomycosis can be histologically separated from other fungi of the gut such as mucormycosis by the presence of angioinvasion, extensive necrosis, and histologically different granulomas with no eosinophil in the latter $[22,23]$.

The other fungal infections with similar morphology to $B$. ranarum are Conidiobolus coronatus, Conidiobolus incongruous, and Pythium insidiobolus (mean hyphal diameter, $4 \mu \mathrm{m}$; ranging in diameter from 2.0 up to $7.0 \mu \mathrm{m}$ ). Both Conidiobolus (mean of the hyphal diameter, $8 \mu \mathrm{m}$; ranging in diameter from 5.0 up to $13 \mu \mathrm{m}$ ) and Basidiobolus species are usually encountered in the materials of the decaying fruit or vegetable and the soil [10]. They are separated from $B$. ranarum by the fact that these fungi affect the head and neck region in immunocompromised patients [20]. Visceral involvement by these fungi has not been reported $[10,24]$. Hussein et al previously examined the ultrastructural features of the fungal elements in basidiobolomycosis. They indicated that the presence of macrophageladen crystalloids, spores, and fungal hyphae [2]. Infection with $B$. ranarum can be detected using immunodiffusion but its sensitivity is controversial. Some studies indicated the utility of molecular techniques (PCR) in the diagnosis of basidiobolomycosis. The best results were obtained by using DNA extracted from formalin-fixed paraffin-embedded lesional tis- sues. However, this method is not widely used by many health institutions due to the rarity of basidiobolomycosis infections $[8,21-23,25,26]$.

Geramizadeh et al reviewed the cases of GIB that have been reported in the English literature from 1964 through 2013. The age of the patients ranged from 2.0 years up to 80 years old. The disease was more common in males, and almost all the patients presented with abdominal pain and fever with a high ESR and counts of eosinophils. Radiological findings included the thickening of the walls of the intestine or stomach. All the preoperative diagnostic studies (radiology and colonoscopy) failed to establish the diagnosis. The management options in most cases were surgery and antifungal drugs [10]. The delay in management and disseminated disease resulted in the death of eight cases. Five cases had recurrence despite combined surgery and antifungal treatment $[11,27]$. The delayed management of GIB leads to several complications such as bowel obstruction, perforation, the formation of a fistulous tract with the adjacent organs, and distant dissemination which is life-threatening [3]. The late presentation of GIB in the cases described by our study is because the preoperative diagnosis of GIB by clinical and radiological findings is impossible for several reasons. Clinically, GIB involves immunocompetent individuals [28], and thus, it is very difficult for the physicians to think about this possibility in a completely healthy patient [27].

Radiologically, the findings of GIB such as diffuse wall thickening and mass lesions are nonspecific and can be encountered in several conditions such as inflammatory bowel diseases and malignant tumors [29]. Similarly, colonoscopic biopsies are usually non-representative or nonspecific in GIB because the fungal elements (B. ranarum) usually grow deep in the submucosa and muscle layers [30]. It should be empha- 

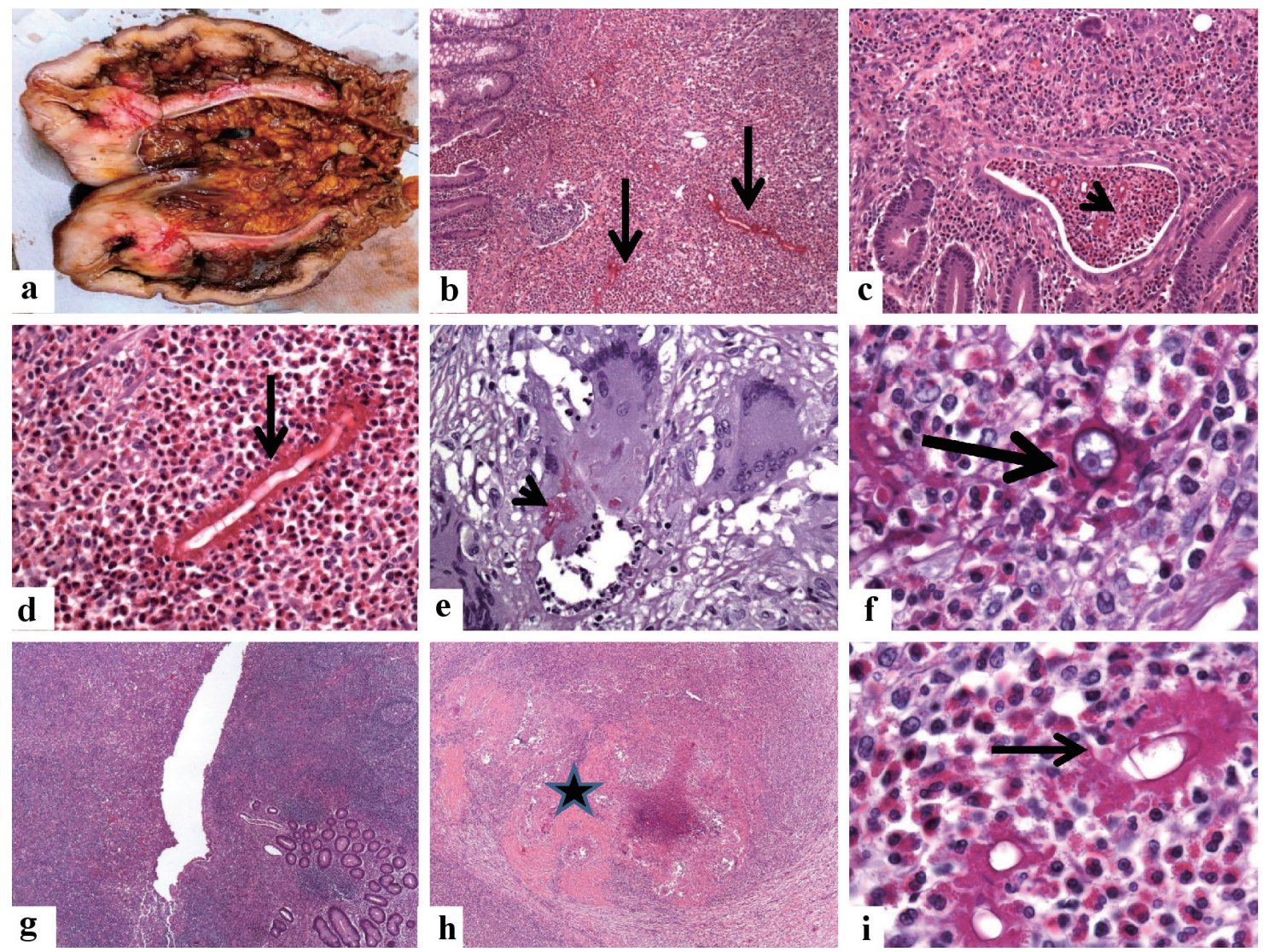

Figure 7. Pathological features of the cecal and appendicular basidiobolomycosis (case 2). (a) En bloc resection of the terminal ileum, appendix, cecum, and ascending colon. There is marked diffuse thickening of the wall of the cecum extending into the proximal part of the ascending colon with obliteration of the lumens. (b-d) The microscopy of the cecal mass reveals a transmural inflammation involving the mucosa (arrow, b: $\times 100$ ) with the formation of eosinophil crypt abscess (arrowhead, c: $\times 40$ ), submucosa, of the cecum. The inflammatory reaction consists of eosinophil-rich mixed inflammation contains fungal hyphae surrounded by the eosinophilic Splendore-Hoeppli bodies (d: $\times 200$, black arrow). The Basidiobolus hyphae are thin-walled, septated hyphae, surrounded by eosinophilic material. (e, f) Granulomatous reactions with the formation of giant cells hosting some fungal structures are seen (PAS stain, arrowhead, e: $\times 400$ ). The zygospores resemble trophozoites of amoeba, have foamy cytoplasm with a large nucleolus but no heterochromatin ( $\mathrm{f}: \times 1,000$, arrowhead), amid eosinophilic microabscess. $(\mathrm{g}-\mathrm{i})$ Sections from the appendix $(\mathrm{g}: \times 40)$ show transmural necrotizing inflammation (star, $\mathrm{h}: \times 40)$, composed of eosinophil-rich inflammatory cell infiltrate. The fungal elements are surrounded by the eosinophilic Splendore-Hoeppli bodies (i: $\times 1,000$, thin arrow).

sized that once GIB is suspected, colonoscopic tissue sampling (biopsies) of the suspected lesions should be performed. If the diagnosis is established based on culture, morphology, or PCR, then empirical treatment with strong antifungal agents should be given to the patients without delay to avoid the bad outcome of this life-threatening condition.

The molecular findings have provided a better understanding of the speciation of Zygomycetes in culture and both formalin-fixed and frozen tissue specimens [31]. The identification of the species of Zygomycetes from cultures has been made feasible using several target loci and molecular techniques [32-37]. The target loci include 28S (large subunit ribosomal DNA), $18 \mathrm{~S}$ (small subunit ribosomal DNA), ribosomal DNA internal transcribed spacer (ITS), cytochrome $b$ gene (Cyt $b$ ), high-affinity iron permease 1 gene (FTR1), genes coding for actin, elongation factor 1-a or lactate dehydrogenase $[31,38-40]$. The methods used in the molecular analysis can be broadly divided into two major categories [41]. The first methods are known as "sequencing-based tests" which report a nucleotide base sequence from a target area followed by sequence comparison analysis. In situ hybridization with pan-fungal and Zygomycetes-specific probes are used. The non-sequencing-based tests use other outcome results for the identification of the species. These approaches include conventional PCR, multiplex PCR using specific DNA probes in a microarray-based assay, real-time PCR using melting curve analysis [39], MicroSeq, semi-nested PCR using Luminex microbead hybridization technology, and restriction fragment length polymorphism (RFLP) [31-40].

To conclude, our study presents a reappraisal of GIB. It also provides further documentation of a primary cecal basidiobolomycosis in healthy immunocompetent adults. Its diagnosis requires considering this rare entity in the differential clinical impressions of immunocompetent individuals having nonspecific clinical, radiological, and endoscopic findings, especially in the endemic areas of basidiobolomycosis. Con- 
firmation of the clinical impression requires tissue sampling (colonoscopic biopsy or surgical resection) for evaluation of the morphological features, together with the use of fungal culture and molecular analysis. The treatment options include immediate intake of antifungal agents and surgical resection. The prompt institution of these therapeutic options can help avoid the dissemination of the fungal structures and their serious lifethreatening complications.

\section{Learning points}

GIB is acquired in most cases by ingestion of contaminated water or food. GIB can affect both immunocompromised and immunocompetent patients. The diagnosis of GIB depends on the detection of B. ranarum on a morphological, microbiological, or molecular basis. The other fungal infections with similar morphology to B. ranarum are Conidiobolus coronatus, Conidiobolus incongruous, and Pythium insidiobolus. On radiology, the findings of GIB are usually nonspecific and may simulate a cancer. On the morphology, GIB has several histological faces that can mimic several disease entities such as granulomatous colitis, diffuse active colitis, inflammatory bowel disease, and lymphoid hyperplasia/eosinophilic colitis. Therefore, the histological findings should be interpreted in the context of the clinical, radiological, and microbiological findings to reach a definitive diagnosis. Empirical treatment with antifungal agents should be given if there is a clinical suspicion of GIB.

\section{Acknowledgments}

None to declare.

\section{Financial Disclosure}

This research did not receive any specific grant from funding agencies in the public, commercial, or not-for-profit sector.

\section{Conflict of Interest}

None to declare.

\section{Informed Consent}

Informed consents were obtained from the patients.

\section{Author Contributions}

All authors certify that he or she has participated sufficiently in the intellectual content and the analysis of data. Each author has reviewed the final version of the manuscript and approved it for publication. Dr. Abdullah S. Alqahtani is the principal investigator responsible for this project.

\section{Data Availability}

The authors declare that data supporting the findings of this study are available within the article.

\section{References}

1. Okafor JI, Testrake D, Mushinsky HR, Yangco BG. A Basidiobolus sp. and its association with reptiles and amphibians in southern Florida. Sabouraudia. 1984;22(1):47-51.

2. Hussein MR, Musalam AO, Assiry MH, Eid RA, El Motawa AM, Gamel AM. Histological and ultrastructural features of gastrointestinal basidiobolomycosis. Mycol Res. 2007;111(Pt 8):926-930.

3. Al-Shanafey S, AlRobean F, Bin Hussain I. Surgical management of gastrointestinal basidiobolomycosis in pediatric patients. J Pediatr Surg. 2012;47(5):949-951.

4. Hassan HA, Majid RA, Rashid NG, Nuradeen BE, Abdulkarim QH, Hawramy TA, Rashid RM, et al. Eosinophilic granulomatous gastrointestinal and hepatic abscesses attributable to basidiobolomycosis and fasciolias: a simultaneous emergence in Iraqi Kurdistan. BMC Infect Dis. 2013;13:91.

5. Bigliazzi C, Poletti V, Dell'Amore D, Saragoni L, Colby TV. Disseminated basidiobolomycosis in an immunocompetent woman. J Clin Microbiol. 2004;42(3):13671369.

6. Al Jarie A, Al Azraki T, Al Mohsen I, Al Jumaah S, Almutawa A, Mohd Fahim Y, Al Shehri M, et al. Basidiobolomycosis: Case series. J Mycol Med. 2011;21(1):37-45.

7. Kian Joe L, Pohan A, Tjoei Eng NI, Van Der Meulen H. Basidiobolus ranarum as a cause of subcutaneous mycosis in Indonesia. AMA Arch Derm. 1956;74(4):378-383.

8. Rabie ME, El Hakeem I, Al-Shraim M, Al Skini MS, Jamil $\mathrm{S}$. Basidiobolomycosis of the colon masquerading as stenotic colon cancer. Case Rep Surg. 2011;2011:685460.

9. Deshpande MV. Mycopesticide production by fermentation: potential and challenges. Crit Rev Microbiol. 1999;25(3):229-243.

10. Geramizadeh B, Heidari M, Shekarkhar G. Gastrointestinal Basidiobolomycosis, a Rare and Under-diagnosed Fungal Infection in Immunocompetent Hosts: A Review Article. Iran J Med Sci. 2015;40(2):90-97.

11. Vikram HR, Smilack JD, Leighton JA, Crowell MD, De Petris G. Emergence of gastrointestinal basidiobolomycosis in the United States, with a review of worldwide cases. Clin Infect Dis. 2012;54(12):1685-1691.

12. Geramizadeh B, Modjalal M, Nabai S, Banani A, Forootan HR, Hooshdaran F, Hooshdaran F, et al. Gastrointestinal zygomycosis: a report of three cases. Mycopathologia. 2007;164(1):35-38.

13. Rose SR, Lindsley MD, Hurst SF, Paddock CD, Damodaran T, Bennett J. Gastrointestinal basidiobolomycosis treated with posaconazole. Med Mycol Case Rep. 2012;2:11-14.

14. Saadah OI, Farouq MF, Daajani NA, Kamal JS, Ghanem AT. Gastrointestinal basidiobolomycosis in a child; an 
unusual fungal infection mimicking fistulising Crohn's disease. J Crohns Colitis. 2012;6(3):368-372.

15. Ejtehadi F, Anushiravani A, Bananzadeh A, Geramizadeh B. Gastrointestinal basidiobolomycosis accompanied by liver involvement: a case report. Iran Red Crescent Med J. 2014;16(9):e14109.

16. Khan ZU, Khoursheed M, Makar R, Al-Waheeb S, AlBader I, Al-Muzaini A, Chandy R, et al. Basidiobolus ranarum as an etiologic agent of gastrointestinal zygomycosis. J Clin Microbiol. 2001;39(6):2360-2363.

17. El-Shabrawi MH, Kamal NM, Kaerger K, Voigt K. Diagnosis of gastrointestinal basidiobolomycosis: a minireview. Mycoses. 2014;57(Suppl 3):138-143.

18. Lyon GM, Smilack JD, Komatsu KK, Pasha TM, Leighton JA, Guarner J, Colby TV, et al. Gastrointestinal basidiobolomycosis in Arizona: clinical and epidemiological characteristics and review of the literature. Clin Infect Dis. 2001;32(10):1448-1455.

19. Busca A, Marmont F, Locatelli F, Limerutti G, Sorrentino MT, Barbui A, Patrono D, et al. Combined antifungal therapy, iron chelation and surgical resection as treatment of hepatic zygomycosis in a patient with haematological malignancy. Mycoses. 2010;53(3):275-278.

20. Nemenqani D, Yaqoob N, Khoja H, Al Saif O, Amra NK, Amr SS. Gastrointestinal basidiobolomycosis: an unusual fungal infection mimicking colon cancer. Arch Pathol Lab Med. 2009;133(12):1938-1942.

21. Wasim Yusuf N, Assaf HM, Rotowa NA. Invasive gastrointestinal Basidiobolus ranarum infection in an immunocompetent child. Pediatr Infect Dis J. 2003;22(3):281282.

22. Lalwani S, Govindasamy M, Gupta M, Siraj F, Varma V, Mehta N, Kumaran V, et al. Gastrointestinal mucormycosis - four cases with different risk factors, involving different anatomical sites. Indian J Gastroenterol. 2012;31(3):139-143.

23. Goel P, Jain V, Sengar M, Mohta A, Das P, Bansal P. Gastrointestinal mucormycosis: a success story and appraisal of concepts. J Infect Public Health. 2013;6(1):58-61.

24. Zavasky DM, Samowitz W, Loftus T, Segal H, Carroll K. Gastrointestinal zygomycotic infection caused by Basidiobolus ranarum: case report and review. Clin Infect Dis. 1999;28(6): 1244-1248.

25. Imwidthaya $\mathrm{P}$, Srimuang $\mathrm{S}$. Immunodiffusion test for diagnosing basidiobolomycosis. Mycopathologia. 1992;118(3):127-131.

26. Gomez-Munoz MT, Fernandez-Barredo S, Martinez-Diaz RA, Perez-Gracia MT, Ponce-Gordo F. Development of a specific polymerase chain reaction assay for the detection of Basidiobolus. Mycologia. 2012;104(2):585-591.

27. van den Berk GE, Noorduyn LA, van Ketel RJ, van Leeuwen J, Bemelman WA, Prins JM. A fatal pseudo-tumour: disseminated basidiobolomycosis. BMC Infect Dis. 2006;6:140.

28. Hassan MIA, Voigt K. Pathogenicity patterns of mucormycosis: epidemiology, interaction with immune cells and virulence factors. Med Mycol. 2019;57(Supplement_2):S245-
S256.

29. Nguyen BD. CT features of basidiobolomycosis with gastrointestinal and urinary involvement. AJR Am J Roentgenol. 2000;174(3):878-879.

30. Al Jarie A, Al-Mohsen I, Al Jumaah S, Al Hazmi M, Al Zamil F, Al Zahrani M, Al Modovar E, et al. Pediatric gastrointestinal basidiobolomycosis. Pediatr Infect Dis J. 2003;22(11):1007-1014.

31. Dannaoui E. Molecular tools for identification of Zygomycetes and the diagnosis of zygomycosis. Clin Microbiol Infect. 2009;15(Suppl 5):66-70.

32. Machouart M, Larche J, Burton K, Collomb J, Maurer $\mathrm{P}$, Cintrat $\mathrm{A}$, Biava $\mathrm{MF}$, et al. Genetic identification of the main opportunistic Mucorales by PCR-restriction fragment length polymorphism. J Clin Microbiol. 2006;44(3):805-810.

33. Hall L, Wohlfiel S, Roberts GD. Experience with the MicroSeq D2 large-subunit ribosomal DNA sequencing kit for identification of filamentous fungi encountered in the clinical laboratory. J Clin Microbiol. 2004;42(2):622-626.

34. Schwarz P, Bretagne S, Gantier JC, Garcia-Hermoso D, Lortholary O, Dromer F, Dannaoui E. Molecular identification of zygomycetes from culture and experimentally infected tissues. J Clin Microbiol. 2006;44(2):340-349.

35. Abe A, Sone T, Sujaya IN, Saito K, Oda Y, Asano K, Tomita F. rDNA ITS sequence of Rhizopus oryzae: its application to classification and identification of lactic acid producers. Biosci Biotechnol Biochem. 2003;67(8):17251731.

36. Nagao K, Ota T, Tanikawa A, Takae Y, Mori T, Udagawa $\mathrm{S}$, Nishikawa T. Genetic identification and detection of human pathogenic Rhizopus species, a major mucormycosis agent, by multiplex PCR based on internal transcribed spacer region of rRNA gene. J Dermatol Sci. 2005;39(1):23-31.

37. Chakrabarti A, Ghosh A, Prasad GS, David JK, Gupta S, Das A, Sakhuja V, et al. Apophysomyces elegans: an emerging zygomycete in India. J Clin Microbiol. 2003;41(2):783-788.

38. Nyilasi I, Papp T, Csernetics A, Krizsan K, Nagy E, Vagvolgyi C. High-affinity iron permease (FTR1) gene sequence-based molecular identification of clinically important Zygomycetes. Clin Microbiol Infect. 2008;14(4):393-397.

39. Hata DJ, Buckwalter SP, Pritt BS, Roberts GD, Wengenack NL. Real-time PCR method for detection of zygomycetes. J Clin Microbiol. 2008;46(7):2353-2358.

40. Kontoyiannis DP, Lionakis MS, Lewis RE, Chamilos G, Healy M, Perego C, Safdar A, et al. Zygomycosis in a tertiary-care cancer center in the era of Aspergillus-active antifungal therapy: a case-control observational study of 27 recent cases. J Infect Dis. 2005;191(8):1350-1360.

41. Spiess B, Seifarth W, Hummel M, Frank O, Fabarius A, Zheng C, Morz H, et al. DNA microarray-based detection and identification of fungal pathogens in clinical samples from neutropenic patients. J Clin Microbiol. 2007;45(11):3743-3753. 